\title{
THE DEFICIENCY INDEX PROBLEM FOR ORDINARY SELFADJOINT DIFFERENTIAL OPERATORS
}

\section{BY ALLEN DEVINATZ ${ }^{1}$}

1. Introduction. In this lecture I shall attempt to survey the development during the past few years of the deficiency index theory for real selfadjoint ordinary differential operators. I shall not attempt to prove any theorems in detail since the proofs tend to be rather technically complicated. Indeed, I shall state only a very few theorems, either formally or informally, since the precise statements of many of the theorems in this field also tend to be rather technically complicated and in some cases rather lengthy. This state of affairs is, of course, not unusual when one is attempting to describe methods for the actual computation of real numbers, in this case nonnegative integers, in contrast, say, to existence or uniqueness statements. However, the ideas underlying many of the theorems and their proofs are often quite simple and I shall outline some of the ways in which the problem has been approached.

The deficiency index problem for ordinary selfadjoint differential operators, at least in the form that we now identify it, goes back to Hermann Weyl [55] around 1910, although in one guise or another it is present in investigations of selfadjoint boundary value problems going back a good deal longer. The work of Weyl as well as subsequent work indicates that there may be close connections between the deficiency index problem and the problem of describing the spectrum, at least qualitatively, of the selfadjoint extensions of the minimal operator associated with a formal ordinary selfadjoint differential operator. In some instances this connection is quite explicit in that knowledge of the deficiency index will give qualitative information about the spectra of selfadjoint extensions, and conversely. In other instances the connection takes a less explicit form in that the methods used to investigate one problem can also be used to investigate the other problem. Whether or not there are deeper underlying connections between these problems is not clear. I shall not attempt to deal with spectral problems here since this would take us too far afield, but shall direct my attention exclusively to the narrower problem indicated in the title.

\footnotetext{
An invited address delivered to the 693rd meeting of the Society, March 31, 1972, in St Louis, Missouri; received by the editors May 20, 1973.

AMS (MOS) subject classifications (1970). Primary 34B20.

Key words and phrases. Selfadjoint ordinary differential operators, deficiency index.

${ }^{1}$ Partially supported by NSF Grant GP-27950.
} 
2. The problem. Perhaps the best way to begin our discussion is by stating the problem. Before we do this, however, it would be well to recall some of the basic facts about real formally selfadjoint differential operators. I shall ask the experts to bear with me.

We shall consider an ordinary differential operator

$$
l(y)=\sum_{k=0}^{n}(-1)^{k}\left(p_{n-k} y^{(k)}\right)^{(k)}
$$

defined on some interval $(a, b)$ of the real axis. We suppose that the coefficients $p_{k}$ are real, $p_{0}>0$, and, for the time being, as many times differentiable as we may require. The formal operator (2.1) is selfadjoint in the sense that for any test functions $u, v \in C_{0}^{\infty}(a, b)$

$$
\int_{a}^{b} l(u) \bar{v}=\int_{a}^{b} u \overline{l(v)}
$$

Hence, when restricted to the test functions, $l$ is a densely defined symmetric operator $L_{0}^{\prime}$ in the Hilbert-Lebesgue space $L^{2}(a, b)$ and so has a symmetric closed extension designated by $L_{0}$ and called the minimal operator associated with $l$. The operator $L=L_{0}^{*}$ is called the maximal operator associated with $l$ and is a closed operator which is the restriction of $l$ to those $u \in L^{2}(a, b)$ whose derivatives up to order $(2 n-1)$ exist, $u^{(2 n-1)}$ is locally absolutely continuous, and $l(u) \in L^{2}(a, b)$.

The formal selfadjoint operators which arise from physical problems usually come equipped with natural boundary conditions. If these boundary conditions are of the correct type and there are a correct number of them, then the restriction of $L$ to those elements in its domain which satisfy the boundary conditions is a selfadjoint operator $H$ which satisfies the inclusion relation $L_{0} \subseteq H \subseteq L$. Since $H$ is selfadjoint it has a spectral expansion. This spectral expansion allows us to obtain quantities which are of physical significance as well as to expand elements of $L^{2}(a, b)$ in terms of generalized eigenfunctions of the operator $l$.

In order to be a little more specific about the boundary conditions we may write Green's formula for any two elements $u$ and $v$ in the domain $D(L)$ of $L$ as

$$
\int_{a}^{b} L(u) \bar{v}-u \overline{L(v)}=[u, v](b)-[u, v](a),
$$

where for each $t \in(a, b),[u, v](t)$ is a sesquilinear form on $D(L) \times D(L)$ which is a differential operator in $u$ and $v$ of order $2 n-1$, and

$$
[u, v](b)=\lim _{t \uparrow b}[u, v](t), \quad[u, v](a)=\lim _{t \downarrow a}[u, v](t) .
$$

Thus each $v \in D(L)$ may be considered as a linear functional acting on 
$D(L)$ which vanishes on $D\left(L_{0}\right)$. Conversely, any linear functional on $D(L)$ which vanishes on $D\left(L_{0}\right)$ may be identified with an element of $D(L)$, uniquely modulo $D\left(L_{0}\right)$. Any element of $D(L)$ is called a linear boundary value for $L$ or $L_{0}$, and a set of linear boundary values is said to be linearly independent if the set is linearly independent when its elements are considered as linear functionals.

In von Neumann's theory of the extension of symmetric operators two cardinal numbers play a vital role. For the case of the minimal operator $L_{0}$, defined above, these are the numbers

$k=\operatorname{dim}\left\{\left(L_{0}-\bar{z} I\right) D\left(L_{0}\right)\right\}^{\perp}, \quad m=\operatorname{dim}\left\{\left(L_{0}-z I\right) D\left(L_{0}\right)\right\}^{\perp}, \quad \operatorname{Im} z>0$.

Each cardinal is called a deficiency number and the pair $(k, m)$ is called the deficiency index of $L_{0}$. As is well known $L_{0}$ is selfadjoint if and only if $k=m=0$; otherwise $L_{0}$ has selfadjoint extensions if and only if $k=m$, and there are an infinite number of such extensions if $m \neq 0$.

Since the differential operator $l$ of $(2.1)$ has real coefficients the operator $L_{0}$ permutes with the ordinary conjugation operator. Hence $k=m$ so that the operator $L_{0}$ has selfadjoint extensions. Thus we may formulate the deficiency index problem in the following way.

DEFICIENCY INDEX PROBLEM. Find the dimension of the linear space of square integrable solutions to the equation

$$
l(y)=z y, \quad \operatorname{Im} z \neq 0 .
$$

The problem itself is certainly easy enough to state and to understand. But to obtain the solution to it is quite another matter, and even for many low order operators with explicitly given coefficients of a simple nature we do not know how to do it. However, the situation is by no means hopeless, and in the last half decade or so some general theorems have emerged which have begun to give some semblence of order to the theory.

Aside from the reasons given in the introduction there is another reason why the solution of the problem is of interest. This is that the deficiency number is the same as the number of linearly independent selfadjoint boundary conditions needed in order to get a selfadjoint extension of $L_{0}$. In order to explain this a little more fully let us call a linearly independent set $B$ of boundary values for $L_{0}$ symmetric if for every $u, v$ in $B,[u, v](a)=[u, v](b)$. If we set

$$
D=\{u: \forall v \in B,[u, v](a)=[u, v](b)\},
$$

and $H=L \mid D$, then it turns out that $H^{*}$ is a symmetric extension of $L_{0}$. Conversely, every symmetric extension of $L_{0}$ may be obtained from a symmetric set of boundary values in this way. A maximal symmetric set of boundary values for $L_{0}$ is called a selfadjoint set of boundary values for $L_{0}$ 
and these sets give rise to selfadjoint extensions of $L_{0}$. Conversely, every selfadjoint extension of $L_{0}$ comes from a selfadjoint set of boundary values. Thus the deficiency number is the same as the number of elements in any selfadjoint set of boundary values. However, perhaps above and beyond any of the reasons given previously, the problem is interesting because it is difficult, and therefore challenging.

The deficiency index problem can be simplified somewhat and this turns out to be rather useful. First of all we notice that by the existence theory for ordinary differential equations the deficiency number does not exceed the order of the differential operator (which must be even). However, considerably more can be said.

The formal differential operator is called regular if $(a, b)$ is a finite interval and $1 / p_{0}, p_{1}, \ldots, p_{n}$ are integrable on this interval. In this case the existence theory for ordinary linear differential systems tells us that the dimension of the linear space of square integrable solutions to the equation (2.4) is $2 n$. The operator is called singular if it is not regular. The operator $l$ is called regular at the endpoint $a$ if $a$ is finite and if, for every $c$, $a<c<b$, the operator $l_{c}$ obtained from $l$ by restricting the coefficients to $(a, c)$ is regular. With one regular endpoint the deficiency number $m$ always satisfies the inequality $n \leqq m \leqq 2 n$. Moreover, as I. M. Glazman [26], [27] showed in 1949, given any $m$ in that range it is always possible to find a selfadjoint differential operator having the deficiency number $m$. He thus corrected some misstatements which had appeared earlier in the literature.

Suppose that $c \in(a, b)$. Let $l^{-}$and $l^{+}$be the differential operators obtained from $l$ by restricting the coefficients of $l$ to $(a, c)$ and $(c, b)$, respectively. If $\mathrm{m}^{-}$and $\mathrm{m}^{+}$are the corresponding deficiency numbers, then the deficiency number $m$ of $L_{0}$ can be shown to be (see [27], [36], [39])

$$
m=m^{-}+m^{+}-2 n .
$$

Consequently, in order to be able to compute the deficiency index of any selfadjoint differential operator, it is enough to be able to compute the deficiency index of an operator which is regular at one endpoint. Hence, from this point on we shall assume that our differential operators are regular at one endpoint.

3. The asymptotic method. Since 1910 , when Weyl's work appeared, until about the mid 1940's only a few scattered papers appeared dealing with the theory of selfadjoint boundary value problems. In the mid and latter forties the work was taken up again, mainly in England, Russia and the United States. In England, and in the United States, the specific problem of the deficiency index was studied only for second order 
operators, while in Russia the beginnings were made on the establishment of a theory for higher order operators. Workers in the United States especially established a large number of criteria for computing the deficiency index of second order operators. A fairly complete list of such results may be found in [12, Chapter 13] where also an outline of the history of the development of the subject may be found.

Most of the theorems for second order operators are concerned with giving a sufficient condition on the coefficients in order that an operator be in the limit point case; that is to say, its deficiency index is $(1,1)$. Since a second order operator, regular at one endpoint, must have deficiency index $(1,1)$ or $(2,2)$, in order to show that the operator is in the limit point case it is sufficient to show that there exists a solution to (2.4) which is not square integrable. Trying to extend this idea to operators of higher order usually presents serious difficulties and more round about methods must be sought.

With the exception of second order and in some cases fourth order operators, the only known method whereby one may identify a singular differential operator whose deficiency index is not half the order of the operator is by the asymptotic method. By this we mean that one tries to obtain easily handled asymptotic estimates for the rate of growth of a complete set of linearly independent solutions to the equation (2.4). Knowing the rate of growth we can then usually tell the dimension of the space of square integrable solutions to this equation.

The Russian school used the asymptotic method in the early 1950's to get deficiency index theorems for higher order operators. In 1953 S. A. Orlov [41] (see also [40]) using a very special class of coefficients was able to confirm Glazman's result that the deficiency number (for differential operators with one regular endpoint) could take on any value between $n$ and $2 n$. M. A. Naimark [39] looked at this problem for operators of arbitrary even order $2 n$, and for a number of different classes of general coefficients. He was able to find one general class of coefficients for which the deficiency number of each corresponding operator is $n+1$. However, for the other classes of coefficients that he investigated it turned out each corresponding operator has deficiency number $n$.

The asymptotic method in the deficiency index problem can be brought to a certain stage of generality by using an asymptotic theorem of $\mathrm{N}$. Levinson [37], [4, p. 92] and certain extensions of it [10], [24]. The scope of Levinson's theorem, as applied to these problems, can be very much broadened if, instead of trying to apply it directly to the given differential operator, one makes certain transformations on the independent and dependent variables. This is, of course, an old idea in the theory of differential equations. The deficiency index theorem which one gets in this 
way [5], [6] cannot be stated in a short and succinct way. It is rather a long prescription on how to compute the deficiency number when the coefficients satisfy certain conditions. However, the underlying idea is quite simple and this can be easily described.

Before explaining the underlying idea let us describe a more general operator than an ordinary differential operator. This is a quasi-differential operator. It has the advantage that a theory can be developed without making any differentiability assumptions about the coefficients. Hence in some respects the theory is simpler. Let $\left\{p_{k}: 0 \leqq k \leqq n\right\}$ be a set of Borel measurable functions defined on the interval $(a, b)$. The quasi-derivatives of a function defined on this interval, with respect to the functions in the class $\left\{p_{k}\right\}$ are, provided they exist, given by

$$
\begin{aligned}
y^{[k]} & =y^{(k)}, & & 1 \leqq k \leqq n-1, \\
y^{[n]} & =p_{0} y^{(n)}, & & \\
y^{[n+k]} & =p_{k} y^{(n-k)}-(d / d t) y^{[n+k-1]}, & & 1 \leqq k \leqq n .
\end{aligned}
$$

By these expressions we mean that the $y^{[k]}$ are locally absolutely continuous for $0 \leqq k \leqq n-1$, and $p_{k} y^{(n-k)}$ and $y^{[n+k]}$ are locally absolutely continuous for $0 \leqq k \leqq n-1$. The quasi-differential operator $l(y)$ is taken to be $y^{[2 n]}$. It is not hard to show that the set of functions whose quasiderivatives exist and vanish off of compact subintervals of $(a, b)$ is dense in $L^{2}(a, b)$ and hence a minimal operator $L_{0}$ and a maximal operator $L$ can be defined. The remainder of the theory of boundary value problems goes forward in virtually the same way as in the case of ordinary differential operators. We refer the reader to either [27] or [39] for an excellent development of the basic ideas.

The matrix differential equation corresponding to the quasi-differential operator $l(y)-z y=y^{[2 n]}-z y$ is

$$
d u(t) / d t=B(t) u(t)
$$

where the matrix $B$ is given by

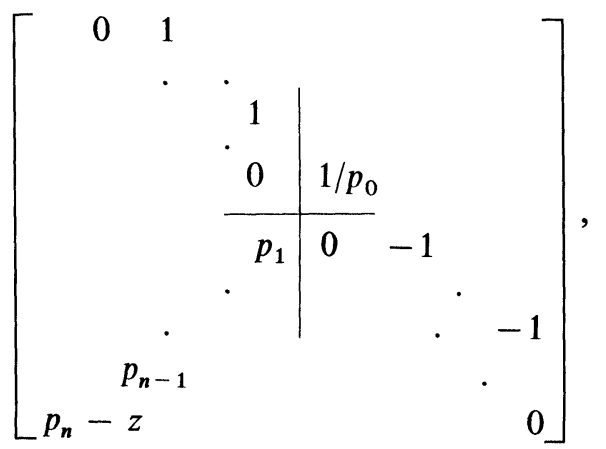


and where all unmarked entries are zero. The quasi-differential operator $y^{[2 n]}-z y$ and the system (3.1) are equivalent in the sense that if $y^{[2 n]}-z y=0$, then $u=\left(y, y^{[1]}, \ldots, y^{[2 n-1]}\right)$ is a solution of $(3.1)$ and if $u=\left(u_{0}, u_{1}, \ldots, u_{n-1}\right)$ is a solution of (3.1), then $y=u_{0}$ is a solution to $y^{[2 n]}-z y=0$ and $y^{[k]}=u_{k}$ for $0 \leqq k \leqq 2 n-1$.

For the sake of convenience let us suppose that we are working on $(0, \infty)$. Let $Q_{0}$ be a nonnegative Borel measurable function on $(0, \infty)$ so that $1 / Q_{0}$ is integrable on every finite interval $(0, t)$, but is not integrable over $(0, \infty)$. Let us set $s(t)=\int_{0}^{t} Q_{0}^{-1}$. The function $s(t)$ is a monotone increasing, locally absolutely continuous function, and has a monotone increasing inverse which we designate by $t=t(s)$. If we set $v(s)=u(t(s))$, then we get from (3.1)

$$
d v(s) / d s=Q_{0}(t(s)) B(t(s)) v(s) .
$$

Let $Q_{1}, \ldots, Q_{n}$ be positive functions on $(0, \infty)$ which are locally absolutely continuous. Let $Q$ be the diagonal matrix

$$
Q=\operatorname{diag}\left[Q_{n}, \ldots, Q_{1}, Q_{1}^{-1}, \ldots, Q_{n}^{-1}\right]
$$

and set $v(s)=Q(t(s)) w(s)$. Then (3.2) becomes

$$
d w(s) / d s=C(s) v(s),
$$

where the matrix $C$ is given by

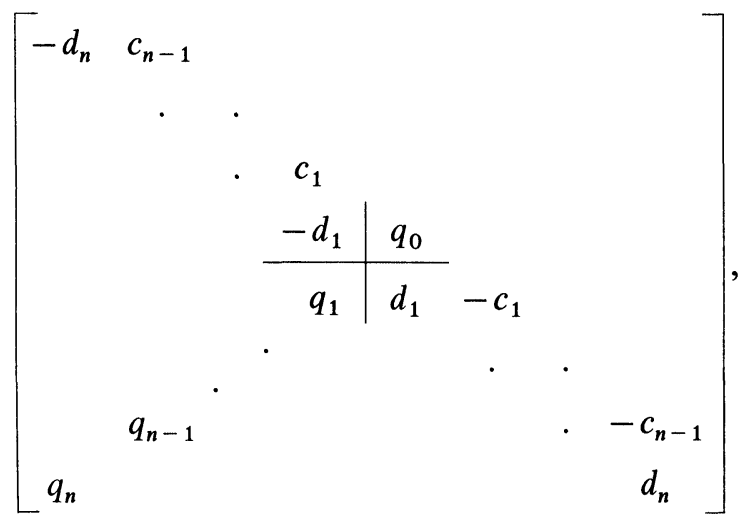

$$
\begin{aligned}
& q_{0}=Q_{0} / Q_{1}^{2} p_{0} \\
& q_{k}=Q_{0} Q_{k}^{2} p_{k}, \quad 1 \leqq k \leqq n-1, \\
& q_{n}=Q_{0} Q_{n}^{2}\left(p_{n}-z\right) \text {, } \\
& d_{k}=\left(d Q_{k} / d s\right) / Q_{k}=Q_{0}\left(d Q_{k} / d t\right) / Q_{k}, \quad 1 \leqq k \leqq n, \\
& c_{k}=Q_{0} Q_{k} / Q_{k+1}, \quad 1 \leqq k \leqq n-1 \text {, }
\end{aligned}
$$


the other entries of $C$ are zero, and all functions have been evaluated at $t(s)$.

We now assume we can write

$$
C(s)=A+V(s)+R(s),
$$

where $A$ is a constant matrix with simple eigenvalues, $V(s) \rightarrow 0$ as $s \rightarrow \infty$, and $V^{\prime}(s)$ and $R(s)$ are integrable in some interval $(a, \infty), a \geqq 0$. With these hypotheses and under certain suitable hypotheses on the characteristic roots $\left\{\lambda_{k}(s)\right\}$ of $A+V(s)$, for example, $\operatorname{Re}\left[\lambda_{j}(s)-\lambda_{k}(s)\right]$ does not change sgn for all sufficiently large $s$, the asymptotic theorem of Levinson can be applied. This says that there is a complete set $\left\{w_{k}\right\}$ of solutions to (3.4) and an $s_{0}>0$ so that $w_{k}(s) \exp -\int_{s_{0}}^{s} \lambda_{k} \rightarrow e_{k}$, where $\left\{e_{k}\right\}$ is a complete set of eigenvectors for $A$. This gives an estimate on the growth of $w_{k}$ and transforming back we get estimates on the growth of a complete set of solutions to (2.4).

The asymptotic method reduces the deficiency index problem to the computation or estimation of the roots of a characteristic polynomial. Although the latter problem is in itself not always simple, nevertheless if it is agreed that this is a reasonable reduction of the deficiency index problem, then a certain plateau of generality has been reached. By suitably choosing the functions $Q_{0}, Q_{1}, \ldots, Q_{n}$ and slightly extending Levinson's theorem it is possible to get all of the deficiency index theorems, obtained by asymptotic methods, which appeared in the literature up until 1971. Moreover, the general theorem obtained by applying the ideas we have described above is psychologically satisfying in the sense that it places all of the special theorems which had been obtained previously into a general framework and subsumes under general principles the sometimes laborious computations required to obtain the special cases. Further, using the general theorem, it is very easy to obtain any number of examples which verify Glazman's result that the deficiency number of a selfadjoint operator, regular at one endpoint may take on any value between $n$ and $2 n$.

Asymptotic estimates for solutions of selfadjoint quasi-differential operators are also very useful in obtaining qualitative information about the spectra of selfadjoint extensions of the minimal operator. This was pointed out by I. M. Rapaport [44] and was further developed by M. A. Naimark [39]. The general idea behind this is that estimates on the growth of solutions give estimates on the growth of Green's function.

Unfortunately, it is very easy to give examples of quasi-differential operators for which the asymptotic method is not applicable. Generally speaking when the coefficients have "large" oscillations it is not possible to transform the problem into one which is a small perturbation of a 
differential operator with constant coefficients. And even if such a transformation is possible, the constant matrix $A$ of the decomposition (3.5) may have multiple eigenvalues. The problem of finding asymptotic estimates in the latter case is not an easy one and only recently have some reasonable beginnings been made $[\mathbf{7}],[\mathbf{1 1}],[\mathbf{5 1}],[\mathbf{5 3}]$.

4. Other methods. Since the class of selfadjoint quasi-differential operators represent such a rich diversity of coefficients, in order to try to achieve further progress in the deficiency index problem, the best tactic seems to be to retreat to the investigation of low order operators. Since it appears highly unlikely that the further investigation of second order operators will yield any insights in how to proceed for higher order operators, it seems that the best bet is to begin with fourth order operators. However, results for second order operators may serve as a useful guide to the questions which can be raised in the fourth order case, and in the last few years a number of papers have appeared on the fourth order case which generalize known results for the second order case.

Weyl's original deficiency index result $[55$, p. 238] is that if $p \geqq 0$ on $(0, \infty)$ (or more generally bounded below), then the differential operator

$$
-y^{(2)}+p y
$$

has deficiency index $(1,1)$. In 1949, Hartman and Wintner [30], and Titchmarsh [48], working independently, showed that if $K$ is a positive constant and $p(t) \geqq-K t^{2}$ then the deficiency index of the operator (4.1) is still $(1,1)$. The exponent 2 is best possible in the sense that if it is replaced by $2+\varepsilon, \varepsilon>0$, the result may no longer be true.

In 1968-1969, W. N. Everitt [20], [21] obtained analogous results for the class of fourth order differential operators

$$
y^{(4)}-\left(p_{1} y^{(1)}\right)^{(1)}+p_{2} y .
$$

He proved that the deficiency number is one-half the order of the operator, i.e. the deficiency index is $(2,2)$, under either of the following conditions:

(i) $p_{2} \geqq 0$ and $0 \leqq p_{1} \leqq K t^{2}\left(1+p_{2}\right)^{1 / 2}, K>0$;

(ii) $p_{2} \geqq-K t^{4 / 3}$ and $\left|p_{1}(t)\right| \leqq K t^{2 / 3}, K>0$.

He was able to establish for case (ii) that $4 / 3$ is the best possible exponent in the lower bound for $p_{2}$, and it was later established by M. Eastham [14] that $2 / 3$ is the best possible exponent in the bound for $p_{1}$.

The natural question raised by case (i) of Everitt's results was whether or not the bounds obtained in this case were also best possible. It seemed that the easiest way to test this was to try to compute the deficiency numbers for a class of fourth order operators with well-behaved 
coefficients, for example the class of operators

$$
y^{(4)}-a\left(t^{\alpha} y^{(1)}\right)^{(1)}+b t^{\beta} y,
$$

where $\alpha, \beta, a$, and $b$ are any real numbers. Note that if we want to insure that the left endpoint is regular for these operators for all $\alpha$ and $\beta$ it is necessary to restrict the coefficient functions to $(1, \infty)$, or else change the coefficients on the interval $(0,1]$, to make the operators regular at zero. This will not change the deficiency index if the operator is already regular at zero.

On the surface it appears that the computation of the deficiency numbers for the class of operators (4.3) should be merely an exercise using the asymptotic method as outlined in \$3. Surprisingly, it turns out to be a rather difficult exercise, and the problem has not yet been completely settled for all values of $\alpha$ and $\beta$. The trouble is that for a large region in the $\alpha, \beta$-plane the matrix $A$ in the decomposition (3.5) does not have simple characteristic roots. There are, as yet, no sufficiently general asymptotic theorems to be of help in such a situation.

The first asymptotic theorem which proved to be useful in the computation of the deficiency numbers of some members of the class (4.3), when the matrix $A$ of (3.5) does not have simple characteristic roots, was obtained by P. W. Walker [50], [51] for fourth order quasi-differential operators. Although his theorem yields considerably more than we shall state here, his results show that in the region $\alpha>2, \beta<\alpha-2$, the deficiency index of (4.3) is 2 when $a>0$ and 3 when $a<0$, regardless of the $\operatorname{sgn}$ of $b$. This already shows that Everitt's result in case (i) is not best possible in any sense. The deficiency numbers of (4.3) for almost all of those values of $\alpha$ and $\beta$ which are not covered by Walker's theorem were subsequently given by Devinatz [7] using a refinement of Levinson's technique. The values of $\alpha$ and $\beta$ which are not covered by the latter paper lie along the lines $\alpha=2$, $\beta \leqq 0$ and $\beta=\alpha-2, \alpha>2$. However, the deficiency numbers along the first line can be computed by other techniques, as noted in that paper.

Although the class of operators (4.3) is very specialized it has, nevertheless, served as a valuable model for obtaining more general theorems as well as a good source of counterexamples. Further, the asymptotic methods developed to handle this special class of operators should serve as a valuable guide in the discovery of new general asymptotic theorems for linear systems of differential equations for which the matrix $A$ in (3.5) has multiple characteristic roots.

Recently D. Hinton [32] has succeeded in generalizing one of Everitt's results to operators of arbitrary order $2 n$. I shall state his result in a form which allows direct comparison with the results of Hartman-Wintner, Titchmarsh, and Everitt, although he has proved considerably more. 
The deficiency index of $y^{[2 n]}$ is $(n, n)$ if $p_{0} \equiv 1$ and

$$
\begin{array}{ll}
p_{k}(t)=O\left(t^{4 k /(4 n-2)}\right), & 1 \leqq k \leqq n-1, \\
p_{n}(t) \geqq-K t^{4 n /(4 n-2)}, & K>0 .
\end{array}
$$

There is some preliminary evidence which indicates that the exponents are probably the best possible. Another interesting aspect of Hinton's general result is that it comes very close to containing a general deficiency result due to $\mathrm{N}$. Levinson [38] for second order operators of the form $-\left(p y^{\prime}\right)^{\prime}+q y$. Levinson's result is that if $M$ is a positive differentiable function so that $M^{\prime}=O\left(M^{3 / 2} / p^{1 / 2}\right), q \geqq-M$, and $\int_{a}^{\infty}(p M)^{-1 / 2}=\infty$, then $y^{[2]}$ has the deficiency index $(1,1)$. Hinton's result requires the additional condition that $p^{\prime}=O\left([p M]^{1 / 2}\right)$. Whether or not this additional condition can be removed by a refinement of his technique or whether it can be removed only for second order operators is still not clear.

Hinton's method of proof is based, in part, on an idea used by Everitt and Levinson, although the methods used by the latter authors did not indicate how their results could be extended to higher order operators. Let $[u, v]$ be the sesquilinear form which arises in Green's formula (2.3). This form is given by

$$
[u, v]=\sum_{k=1}^{n}\left\{u^{[k-1]} \bar{v}^{[2 n-k]}-u^{[2 n-k]} \bar{v}^{[k-1]}\right\} .
$$

From the Lagrange identity

$$
l(u) \bar{v}-u \overline{l(v)}=(d / d t)[u, v]
$$

it follows that if $l(u)=z u, l(v)=\bar{z} v$, then $[u, v]^{\prime}=0$ so that $[u, v]$ is identically a constant. If the deficiency number of the minimal operator $L_{0}$ is greater than half the order of $l$, then it can be shown that there exist eigenvectors $u$ and $v$ so that $[u, v] \equiv 1$. Suppose $w$ is a nonnegative weight function on $(a, \infty)$ which is not integrable. On the other hand suppose that it is possible, by putting suitable restrictions on the coefficients of $l$, to show that the square norms with respect to $w$ of all of the quasiderivatives of any eigenvectors $u$ and $v$ are finite. From the form of $[u, v]$ given in (4.5) and the fact there exist eigenvectors of $u, v$ so that $[u, v] \equiv 1$ we get the contradiction

$$
\infty=\int_{a}^{\infty} w=\int_{a}^{\infty}[u, v] w<\infty .
$$

This is the basic program carried out by Hinton. He first makes a transformation of the associated matrix equation very much in the same spirit as is done in $\S 3$, and then uses the conditions (4.4) to get bounds on the quasi-derivatives with respect to the weight function $w=1 / t$.

Using the same basic idea other results have been obtained by $F$. V. 
Atkinson and W. D. Evans [1] for second order operators and by W. D. Evans [18] for fourth order operators. The results in these latter papers require conditions only on a sequence of intervals in $[0, \infty)$ rather than on the whole half-axis, thus allowing the consideration of "wildly oscillating functions." In this connection see also [2], [15], [16], [17] and [29].

On the other side of this same coin, it is known [39, II, p. 78] that if there exist $u, v$ in $D(L)$ so that $\lim _{t \rightarrow \infty}[u, v](t) \neq 0$, then the deficiency number of $L_{0}$ cannot be half the order of the operator. This fact can be exploited to find limit circle criteria for second order operators (see [16]) and in some cases it can be used to compute the deficiency numbers of fourth order operators. However, for higher order operators it is unlikely that this fact by itself can be used to obtain precise results about deficiency numbers.

For second order quasi-differential operators, regular at one endpoint, and having nonnegative coefficients it is quite easy to prove, modulo known general results, that the operator is in the limit point case. It is not known whether or not this result persists for higher order operators, i.e., whether or not a quasi-differential operator, regular at one endpoint, and having nonnegative coefficients has deficiency number which is half the order of the operator. Besides the paper by Everitt mentioned earlier, there have appeared in the past few years a number of other papers dealing with this question for fourth order operators [8], [13], [20], [34]. Although all of these results have not been proved in the same way they all can be handled by a common idea. The idea is to show that any square integrable solution to $y^{[4]}=0$ which has initial values $y(0)=y^{(1)}(0)=0$ must be identically zero. It will then follow that there exist two linearly independent solutions to $y^{[4]}=0$ with the given initial conditions which are not square integrable. Further, no linear combination of these solutions can be square integrable since a linear combination satisfies the same initial conditions as the individual solutions.

If $y$ satisfies the initial value problem of the last paragraph then the Dirichlet form may be written as

$$
\int_{0}^{s}\left\{p_{0}\left|y^{(2)}\right|^{2}+p_{1}\left|y^{(1)}\right|^{2}+p_{2}|y|^{2}\right\}=\bar{y}^{(s)} y^{[3]}(s)+\bar{y}^{(1)}(s) y^{[2]}(s) .
$$

By using the fact that $y$ is square integrable and various bounding conditions on the coefficients $p_{0}, p_{1}$ and $p_{2}$, it can be shown that the right-hand side goes to zero as $s \rightarrow \infty$. But since $p_{0}>0$ a.e. and the other coefficients are nonnegative it follows that $y=0$. Other results, involving coefficients which are not necessarily nonnegative, have also been obtained by this method [9], this paper containing results which generalize some of the results of $[7]$. 
A technique for extending deficiency index results is the method of perturbation. For example, it is a standard result of Hilbert space theory that if $L$ is a symmetric operator and $Q$ is a bounded selfadjoint operator then $L+Q$ has the same deficiency indices as $L$ itself. In terms of differential operators one of the things this says is that if $q$ is a real bounded measurable function on $[a, \infty)$, then the differential operators $y^{[2 n]}$ and $y^{[2 n]}+q y$ have the same deficiency indices. The natural question which arises is whether or not the same result will persist if other perturbations are allowed. For example one might ask the question if $q \in L^{p}(0, \infty)$ for all $p \geqq 1$. This question is not settled even for second order operators, but some recent results along this line are available [42], [43].

One approach to such questions can be made by means of a perturbation theorem due to $\mathrm{F}$. Reillich [46]. An operator $Q$ is said to be relatively bounded with respect to $P$ or $P$-bounded if $D(P) \subseteq D(Q)$ and

$$
\|Q u\| \leqq a\|u\|+b\|P u\|, \quad u \in D(P) .
$$

Rellich's theorem is the following: If $P$ is essentially selfadjoint and $Q$ is symmetric and $P$-bounded with $0 \leqq b<1$, then $P+Q$ is essentially selfadjoint (see also [35, p. 288]).

Suppose the formally selfadjoint operator $l$ given by (2.1) is regular at the origin and that the deficiency index of the minimal operator $L_{0}$ is $(n, n)$. Extend the coefficients of $l$ to the whole axis by setting $p_{0}(t)=1$, $p_{k}(t)=0,1 \leqq k \leqq n$, for $t<0$. This new operator, restricted to acting only on functions with support in the negative half-axis, has deficiency index $(n, n)$. Hence, by the deficiency numbers relation $(2.5)$ it follows that the minimal operator associated with the extended operator is selfadjoint; i.e., the minimal and the maximal operators are the same. Designate this operator by $P^{\prime}$ and the restriction of $P^{\prime}$ to those elements in its domain which vanish off of compact sets by $P$. The operator $P$ is essentially selfadjoint and its closure is $P^{\prime}$.

Let us now suppose that $0<m \leqq n$ and that there exist positive $k$ and $M$ so that

$$
\left\|y^{(m)}\right\| \leqq k\|y\|+M\|P y\|, \quad y \in D(P) .
$$

Let $Q$ be the operator corresponding to the formally selfadjoint operator $q y$, where $q$ is a real measurable function with $q(t)=0$ for $t<0$, and

$$
\sup _{s>0} \int_{s}^{s+1}|q|^{2}<\infty .
$$

It is clear that $D(P) \subset D(Q)$ and further, as is well known (see [28, pp. 167-169]), for every $\varepsilon>0$ there exists a $K>0$ so that $y \in D(P)$ implies

$$
\|Q y\| \leqq \varepsilon\left\|y^{(m)}\right\|+K\|y\| \text {. }
$$


Thus from (4.7) and (4.9) it follows there exist numbers $a$ and $b, 0 \leqq b<1$ so that (4.6) is satisfied. Consequently, we may apply Rellich's theorem which shows that $P+Q$ is essentially selfadjoint.

Let us split the operator $P+Q$ into the direct sum of two operators $(P+Q)^{-}$and $(P+Q)^{+}$, where these are the operators obtained from $P+Q$ by restricting its coefficients to $(-\infty, 0)$ and $(0, \infty)$, respectively. Clearly $(P+Q)^{-}$has the deficiency index $(n, n)$ so that by the relation (2.5) it follows that the operator $(P+Q)^{+}$also has the deficiency index $(n, n)$. We have arrived at the following result:

If the quasi-differential operator l given by (2.1) is regular at the origin and has deficiency index $(n, n)$, if (4.7) is satisfied with $P$ replaced by $L_{0}^{\prime}$, and if $q$ satisfies (4.8), then the minimal operator associated with the quasidifferential operator $l(y)+q y$ has the deficiency index $(n, n)$.

There are a number of different sufficient conditions that one may put on the coefficients of the operator $l$ in order that an inequality of the form (4.7) may be satisfied. For example, if there exists a constant $M$ so that $M p_{0} \geqq 1$, and if $p_{k} \geqq 0,1 \leqq k \leqq n$, then (4.7) is clearly fulfilled when $P$ is replaced by $L_{0}^{\prime}$. More generally if there exists a constant $m$ so that $p_{k} \geqq m$, $1 \leqq k \leqq n$, and if $M p_{0} \geqq 1$, then we have

$$
\|y\|\left\|L_{0}^{\prime} y\right\| \geqq\left(L_{0}^{\prime} y, y\right) \geqq \frac{1}{M}\left\|y^{(n)}\right\|^{2}+m \sum_{k=1}^{n}\left\|y^{(k)}\right\|^{2} .
$$

Now use the well-known fact that, for every $\varepsilon>0$, there is a $K>0$ so that

$$
\left\|y^{(k)}\right\|^{2} \leqq \varepsilon\left\|y^{(n)}\right\|^{2}+K\|y\|^{2}, \quad 1 \leqq k \leqq n-1 .
$$

This combined with the previous inequality gives an inequality of the form (4.7). The two cases we have mentioned here do not exhaust all possibilities and the reader can certainly supply many more.

In case the operator (2.1) is a differential operator then the previous considerations can be used to perturb by differential operators other than the multiplier operator. If we suppose that $p_{k} \in C^{n-k}([0, \infty)), p_{0}>0$, and that the deficiency index of $L_{0}$ in $(n, n)$, then we may extend $L_{0}^{\prime}$ to a differential operator $P$, defined on $C_{0}^{\infty}$ of the whole axis, whose closure is selfadjoint. We further suppose that (4.7) is satisfied for $m \leqq 2 n$. Let us now take functions $q_{k}, 1 \leqq k \leqq m$, which are sufficiently differentiable and satisfy (4.8). We need not assume that the $q_{k}$ are real, but we do assume that the differential operator

$$
\sum_{k=1}^{m} q_{k} y^{(m-k)}
$$

is formally selfadjoint. It is then not hard to show that the $q_{k}$ may be extended to the whole axis so that the expression (4.10) with the extended functions is formally selfadjoint. Indeed, this may be done in such a way 
that if $Q$ is the minimal operator associated with the extended operator (4.10), then the restriction of the coefficients of $P+Q$ to the negative axis gives a differential operator whose deficiency index is $(n, n)$. Since $D(P)$ consists of functions in $C_{0}^{\infty}$, it is clear that $D(P) \subset D(Q)$. The inequality (4.9) is preserved and hence it follows by the same type of reasoning as before, that the minimal operator associated with $l(y)+\sum_{k=1}^{m} q_{k} y^{(m-k)}$ has the deficiency index $(n, n)$.

As an application of the previous considerations we can take $l(y)=$ $(-1)^{n} y^{(2 n)}$, so that if the differential operator

$$
(-1)^{n} y^{(2 n)}+\sum_{k=1}^{2 n-1} q_{k} y^{(2 n-k)}
$$

is formally selfadjoint, and the $q_{k}$ satisfy (4.8), then the minimal operator associated with this differential operator has the deficiency index $(n, n)$.

5. Summing up. We have seen in the previous sections that two general results have evolved during the past few years which allow one to compute the deficiency indices of relatively wide classes of selfadjoint quasidifferential operators with real coefficients. One result is obtained by means of asymptotic methods. With the exception of second order and in some cases fourth order operators, this is the only method now available for computing deficiency numbers which are not half the order of the operator. Generally speaking smoothness properties of the coefficients of the given pseudo-differential operators are not important. but rather the main drawback to applying this technique is that it cannot handle differential operators whose coefficients have "large oscillations." It seems to me that one principal unsettled problem in this area is to find a technique, other than the asymptotic method, for identifying those quasidifferential operators whose deficiency numbers are not half the order of the operator. As of now there has appeared no hint how one would go about doing this, except, as we have mentioned before, for second order and in some cases for fourth order operators.

The other general result now known is Hinton's theorem which we described in the last section. This theorem gives a wide class of coefficients for which the deficiency number of the corresponding operator is half the order of the operator. Evidence from work on fourth order operators as well as some known results on higher order operators indicates that this theorem may somehow be the best possible in the sense that if more liberal growth conditions are allowed for the coefficients the deficiency number of the corresponding operator may no longer be maintained at half the order of the operator. However, the work of Evans [18] on fourth order operators as well as the results in $[1],[2],[15]$ and $\lceil 29]$ on second 
order operators indicates that it may be enough to prescribe growth conditions on a sequence of nonoverlapping intervals and still maintain the result. This would allow for "wildly oscillating" coefficients. A general theorem of this nature is not yet available.

The question of whether or not a quasi-differential operator, regular at the origin, and having nonnegative coefficients has its deficiency number half the order of the operator is still open. It is of course true for second order operators, being Weyl's original theorem.

The general question of how much one may perturb a selfadjoint differential or quasi-differential operator so as to maintain its deficiency index remains by and large unanswered. One might perhaps start by asking the following very concrete question. Suppose $l(y)$ is a selfadjoint quasi-differential operator, regular at the origin, with real coefficients whose minimal operator has deficiency number half the order of the operator. Suppose $q$ is a locally integrable function for which

$$
\sup _{s>0} \int_{s}^{s+1}|q|<\infty .
$$

Is it true that the minimal operator $l(y)+q y$ has the same deficiency index as $l(y)$ ?

Finally, the ultimate problem of giving necessary as well as sufficient conditions on the coefficients of a differential or quasi-differential operator so that it has a given deficiency index appears to be very far from being solved. To show the difficulty and delicacy of this problem we mention a recent result of Eastham and Thompson [17] concerning second order differential operators $-y^{\prime \prime}+p y$. They show that for any given $\varepsilon>0$ there exist two $C^{\infty}$ functions $p_{1}$ and $p_{2}$ on $(0, \infty)$ which differ only on a sequence of intervals of total length at most $\varepsilon$, so that $-y^{\prime \prime}+p_{1} y$ has the deficiency index $(1,1)$ and $-y^{\prime \prime}+p_{2} y$ has the deficiency index $(2,2)$.

\section{REFERENCES}

The following list is not intended to be complete. It consists of articles quoted in the text and recent articles having a very close relationship to the material in text. A much more complete bibliography will appear in the survey article [23].

1. F. V. Atkinson and W. D. Evans, On solutions of a differential equation which are not of integrable square, Math. Z. 127 (1972), 323-332.

2. I. Brinck, Self-adjointness and spectra of Sturm-Liouville operators, Math. Scand. 7 (1959), 219-239. MR 22 \#3844.

3. J. Chaudhuri and W. N. Everitt, On the square of a formally self-adjoint differential expression, J. London Math. Soc. (2) 1 (1969), 661-673. MR 40 \#1814.

4. E. Coddington and N. Levinson, Theory of ordinary differential equations, McGrawHill, New York, 1955. MR 16, 1022.

5. A. Devinatz, The deficiency index of ordinary self-adjoint differential operators, Pacific J. Math. 16 (1966), 243-257. MR 32 \#2688. 
6. A. Devinatz, The deficiency index of a certain class of ordinary self-adjoint differential operators, Advances in Math. 8 (1972), 434-473.

7. - The deficiency index of certain fourth-order differential operators, Quart. J. Math. Oxford Ser. (2) 23 (1972), 267-286.

8. - Positive definite fourth-order differential operators, J. London Math. Soc. (2) 6 (1973), 412-416.

9. - On limit-2 fourth-order differential operators, J. London Math. Soc. (2) 6 (1973).

10. - An asymptotic theorem for systems of linear differential equations, Trans. Amer. Math. Soc. 160 (1971), 353-363. MR 44 \# 545.

11. A. Devinatz and J. Kaplan, Asymptotic estimates for solutions of linear systems of ordinary differential equations having multiple characteristic roots, Indiana Univ. Math. J. 22 (1972), 355-366.

12. N. Dunford and J. Schwartz, Linear operators. II: Spectral theory. Selfadjoint operators in Hilbert space, Interscience, New York, 1963. MR 32 \#6181.

13. M. S. P. Eastham, The limit-2 case of fourth-order differential equations, Quart. J. Math. Oxford Ser. (2) 22 (1971), 131-134. MR 44 \#1867.

14. - On the $L^{2}$ classification of fourth-order differential equations, J. London Math. Soc. (2) 3 (1971), 297-300. MR 42 \# 7976.

15. - On a limit-point method of Hartman, Bull. London Math. Soc. (to appear).

16. - Limit circle differential expressions of the second order with an oscillating coefficient, Quart. J. Math. Oxford Ser. (2) 24 (1973), 257-263.

17. M. S. P. Eastham and M. L. Thompson, On the limit point, limit-circle classification of second-order ordinary differential equations (to appear).

18. W. D. Evans, On non-integrable square solutions of a fourth-order differential equation and the limit-2 classification, J. London Math. Soc. (to appear).

19. W. N. Everitt, Integrable-square solutions of ordinary differential equations, Quart. J. Math. Oxford Ser. (2) 10 (1959), 145-155; II, 13 (1962), 217-220; III, 14 (1963), 170-180. MR 21 \#5766; MR 25 \#5220; MR 27 \#1644.

20. - Some positive definite differential operators, J. London Math. Soc. 43 (1968), 465-473. MR 37 \# 3086.

21. - On the limit point classification of fourth-order differential operators, J. London Math. Soc. 44 (1969), 273-281. MR 38 \# 3498.

22. - - On the limit-circle classification of second order differential expressions, Quart. J. Math. Oxford Ser. (2) 23 (1972), 193-196.

23. - - Integrable-square solutions of ordinary differential equations, View Arch. Wisk. (to appear).

24. M. V. Fedorjuk, Asymptotic methods in the theory of one-dimensional singular differential operators, Trudy Moskov. Mat. Obšč. 15 (1966), 296-345 = Trans. Moscow Math. Soc. 1966, 333-386. MR 34 \#7870.

25. - The asymptotics of solutions to ordinary linear differential equations of the Nth order, Differencial'nye Uravneniya 2 (1966), 492-507 = Differential Equations 2 (1966), 250-258. MR 34 \#446.

26. I. M. Glazman, On the deficiency indices of differential operators, Dokl. Akad. Nauk SSSR 64 (1949), 151-154. (Russian) MR 10, 538.

27. - On the theory of singular differential operators, Uspehi Mat. Nauk 5 (1950), no. 6 (40), 102-135; English transl., Amer. Math. Soc. Transl. (1) 4 (1962), 331-372. MR 13, 254.

28. S. Goldberg, Unbounded linear operators: Theory and applications, McGraw-Hill, New York, 1966. MR 34 \# 580. 
29. P. Hartman, On the number of $L^{2}$-solutions of $x^{\prime \prime}+q(t) x=0$, Amer. J. Math. 73 (1951), 635-645. MR 13, 462.

30. P. Hartman and A. Wintner, A criterion for the non-degeneracy of the wave equation, Amer. J. Math. 71 (1949), 206-213. MR 10, 455.

31. D. Hinton, Asymptotic behavior of the solutions of $\left(r y^{(m)}\right)^{(k)} \pm q y=0$, J. Differential Equations 4 (1968), 590-596. MR 38 \#373.

32. — Limit point criteria for differential equations, Canad. J. Math. 24 (1972), 293-305; II, Canad. J. Math. (to appear).

33. - Solutions of $\left(r y^{(m)}\right)^{(n)}+q y=0$ of class $L_{p}[0, \infty)$, Proc. Amer. Math. Soc. 32 (1972), 134-138. MR 44 \#5546.

34. - Limit point criteria for positive definite fourth-order differential operators, Quart. J. Math. Oxford Ser. (2) (to appear).

35. T. Kato, Perturbation theory for linear operators, Die Grundlehren der math. Wissenschaften, Band 132, Springer-Verlag, New York, 1966. MR 34 \# 3324.

36. K. Kodaira, On ordinary differential equations of any even order and the corresponding eigenfunction expansions, Amer. J. Math. 72 (1950), 502-544. MR 12, 103.

37. N. Levinson, The asymptotic nature of the solutions of linear systems of differential equations, Duke Math. J. 15 (1948), 111-126. MR 9, 509.

38. - Criteria for the limit-point case for 2nd-order linear differential operators, Časopis Pěst. Mat. Fys. 74 (1949), 17-20. MR 11, 248.

39. M. A. Naimark, Linear differential operators, GITTL, Moscow, 1954; English transl., Ungar, New York, 1967. MR 16, 702; MR 41 \# 7485.

40. F. A. Neimark, On the deficiency index of differential operators, Uspehi Mat. Nauk 17 (1962), no. 4 (106), 157-163. (Russian) MR 26 \#388.

41. S. A. Orlov, On the deficiency index of differential operators, Dokl. Akad. Nauk SSSR 92 (1953), 483-486. (Russian) MR 15, 802.

42. W. T. Patula and J. W. W. Wong, An $L^{p}$-analogue of the Weyl alternative, Math. Ann. 197 (1972), 9-28.

43. W. T. Patula and P. Waltman, Limit point classification of second order linear differential equations (to appear).

44. I. M. Rapoport, On the asymptotic behavior of solutions of linear differential equations, Dokl. Akad. Nauk SSSR 78 (1951), 1097-1100. (Russian) MR 13, 461.

45. - On a singular boundary problem for ordinary linear differential equations, Dokl. Akad. Nauk SSSR 79 (1951), 21-24. (Russian) MR 13, 558.

46. F. Rellich, Störgungstheorie der Spektralzerlegung. III, Math. Ann. 116 (1937), $555-570$.

47. H. Stetkaer-Hansen, A generalization of a theorem of Wienholt z concerning essential self-adjointness of singular elliptic operators, Math. Scand. 19 (1966), 108-112. MR 36 \# 520.

48. E. C. Titchmarsh, On the uniqueness of Green's function associated with a second-order differential equation, Canad. J. Math. 1 (1949), 191-198. MR 10, 537.

49. K. Unsworth, Asymptotic expansions and deficiency indices associated with third-order self-adjoint differential operators, Quart. J. Math. Oxford Ser. (2) 24 (1973), 177-188.

50. P. W. Walker, Asymptotics of the solutions to $\left[\left(r y^{\prime \prime}\right)^{\prime}-p y^{\prime}\right]^{\prime}+q y=\sigma y$, J. Differential Equations 9 (1971), 108-132. MR 42 \#4829.

51. - Deficiency indices of fourth order singular differential operators, J. Differential Equations 9 (1971), 133-140. MR 43 \#6498.

52. — - Asymptotics for a class of weighted eigenvalue problems, Pacific J. Math. 40 (1972), 501-510.

53. - Asymptotics for a class of fourth-order differential equations, J. Differential Equations 11 (1972), 321-334. MR 45 \#3864. 
54. P. W. Walker, Weighted singular differential operators in the limit circle case, J. London Math. Soc. 4 (1972), 741-744.

55. H. Weyl, On ordinary differential equations with singularities and the associated expansions of arbitrary functions, Math. Ann. 68 (1910), 220-269.

56. J. S. W. Wong, On $\mathrm{L}_{2}$-solutions of linear ordinary differential equations, Duke Math. J. 38 (1971), 93-97. MR 42 \# 7973.

57. A. D. Wood, Deficiency indices of some fourth-order differential operators, J. London Math. Soc. (2) 3 (1971), 96-100. MR 43 \#2568.

58. A. Zettl, A note on square integrable solutions of linear differential equations, Proc. Amer. Math. Soc. 21 (1969), 671-672.

Department of Mathematics, NorthWestern University, EVAnston, Illinois 60201. 